\section{Angle-stable measurement of patellar position in clinically normal dogs}

\section{James Miles}

Faculty of Health and Medical Sciences, Copenhagen, Denmark

A simple, angle-stable measurement of patellar proximodistal positioning would be of benefit to clinicians, since it would enable comparison of measurements between radiographs taken pre- and postoperatively and would not be dependent on precise angular alignment of the stifle joint.

Two candidate measurements were identified in a vulpine phase one study, and were investigated further using radiographs of 19 healthy stifles joints available at two stifle angulations $\left(98^{\circ} \pm 8^{\circ}\right.$ and $\left.129^{\circ} \pm 11^{\circ}\right)$ from a previously described survey of Greenland sled dogs. Mean body mass was $40 \pm 5 \mathrm{~kg}$.

Measurement 1 was defined as the angle between lines connecting the caudal aspect of the medial tibial plateau with the caudal aspect of the femoral condyles and the distal patellar pole. Measurement 2 was defined similarly, using the distal articular surface of the patella as the third point.
Although the distal patellar pole is simpler to identify, its position may become obscured by entheseophytosis, commonly seen in the patient populations in which these measurements would be of interest. In humans, entheseophytosis can help illuminate the position of the distal articular surface. Radiographs were read once by one observer using ImageJ.

Paired t-tests showed statistically significant $(p<.05)$ decreases in both measurements 1 and 2 from $98^{\circ}$ to $129^{\circ}$, however the mean difference was only $2.1^{\circ}$ (measurement 1) and $1.5^{\circ}$ (measurement 2), representing approximately $0.05^{\circ}$ $0.07^{\circ}$ change per $1^{\circ}$ change in stifle angle. Although neither measurement was perfectly angle-stable, the amount of variation found was probably clinically unimportant. Reference intervals for this breed at $97^{\circ}$ were $50^{\circ}-59^{\circ}$ for measurement 1 (referencing the distal pole) and $44^{\circ}-53^{\circ}$ for measurement 2 (referencing the articular surface). Higher values indicate a more distally positioned patella, and vice versa.

Previous studies have indicated a possible role of breed size in the magnitude of other patellar positioning indices, and this might hold true for these measurements. Further studies in other dog breeds with differing body masses is indicated to establish specific reference intervals, to determine reliability and to decide which of the two measurements shows most promise for use clinically. 